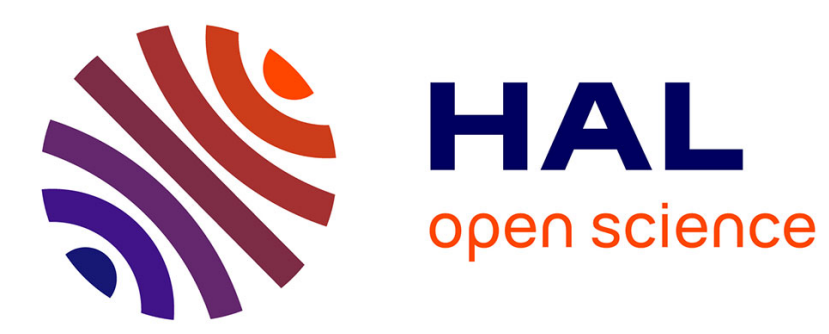

\title{
Adaptation générale de la méthode du diagramme de Nyquist dans le domaine non linéaire
}

A. Blaquière

\section{To cite this version:}

A. Blaquière. Adaptation générale de la méthode du diagramme de Nyquist dans le domaine non linéaire. J. Phys. Radium, 1952, 13 (12), pp.636-644. 10.1051/jphysrad:019520013012063601 . jpa00234663

\section{HAL Id: jpa-00234663 https://hal.science/jpa-00234663}

Submitted on 1 Jan 1952

HAL is a multi-disciplinary open access archive for the deposit and dissemination of scientific research documents, whether they are published or not. The documents may come from teaching and research institutions in France or abroad, or from public or private research centers.
L'archive ouverte pluridisciplinaire HAL, est destinée au dépôt et à la diffusion de documents scientifiques de niveau recherche, publiés ou non, émanant des établissements d'enseignement et de recherche français ou étrangers, des laboratoires publics ou privés. 


\title{
ADAPTATION GÉNÉRALE DE LA MÉTHODE DU DIAGRAMME DE NYQUIST DANS LE DOMAINE NON LINÉAIRE
}

\author{
Par A. BLAQUIÈRE. \\ Laboratoire de Radioélectricité de l’École Normale Supérieure.
}

\begin{abstract}
Sommaire. - On généralise la méthode du diagramme de Nyquist mobile déjà exposée ( ${ }^{1}$ ) sur des exemples simples.

- L'étude précédente est adaptée au cas d'un oscillateur régi par une équation différentielle non linéaire d'un ordre quelconque et l'on détermine à nouveau ses principales caractéristiques :

Loi d'évolution vers un régime stabilisé;

Temps de réaction à une petite perturbation;

Stabilité de fréquence;

Largeur de la plage de synchronisation.

Revenant ensuite aux conditions pour lesquelles la loi d'oscillation est sensiblement linéaire, on est amené à modifier légèrement la représentation classique du diagramme de Nyquist.

Cela fait mieux apparaître la continuité des propriétés physiques de l'oscillateur dans le passage de l'amorçage à la stabilisation, et permet de traiter ce premier cas comme une approximation linéaire du cas général.
\end{abstract}

Nous généraliserons ici la théorie développée dans un précédent exposé $\left({ }^{\mathbf{1}}\right)$. Des exemples simples ont servi jusque-là à en faire comprendre le mécanisme et à montrer qu'elle permet de retrouver tous les résultats classiques.

Nous ne considérerons plus maintenant des modèles particuliers d'oscillateurs, mais nous nous placerons à un point de vue plus général et plus abstrait.

Nous pourrons être amené parfois à répéter l'énoncé de résultats acquis précédemment. Ce sera dans le but de les rassembler sous la forme d'un exposé complet et de permettre au lecteur de se reporter pour plus de détails aux paragraphes antérieurement traités.

1. Pseudo-sinusoïde tangente. - Les oscillateurs à loi non linéaire que nous étudions sont

(1) Les oscillateurs non linéaires et le diagramme deNyquist. caractérisés par le fait que leur oscillation atteint toujours un régime stable à peu près sinusoïdal. Tant qu'ils n'ont pas atteint ce régime, leur évolution est convenablement décrite par une loi pseudosinusoïdale, l'amplitude et la pseudo-période variant lentement avec le temps.

La loi de variation de l'amplitude ne peut plus, dans ce cas, être supposée simplement exponentielle. On doit représenter leur évolution par l'expression plus générale

$$
V=a(t) \sin \omega_{1}(t) t
$$

$a(t)$ étant une fonction caractérisant l'enveloppe de l'oscillation.

Dans un petit domaine, la fonction $a(t)$ peut cependant être assimilée à une exponentielle. On peut, en effet, pour chaque valeur $a_{t_{0}}$ de l'amplitude, trouver facilement une exponentielle tangente à la courbe " $a$ fonction de $t$ ).

On peut trouver, de plus, une pseudo-sinusoïde 
dont l'amplitude varie suivant la loi exponentielle précédente et dont la pseudo-période se confonde, au voisinage de l'instant $t_{0}$, avec la pseudo-période de l'oscillation naturelle.

Nous définissons ainsi la pseudo-sinusoïde tangente au phénomène naturel à un instant $t_{0}$ : à cet instant $t_{0}$, la constante de temps de la pseudo-sinusoïde tangente est confondue avec la constante de temps instantanée pour $t=t_{0}$ de l'oscillation naturelle.

De même, sa pulsation réelle est la pulsation réelle instantanée de l'oscillation naturelle.

Il est possible de grouper en une seule expression complexe la constante de temps et la pulsation réelle et de définir, comme nous l'avons fait antérieurement, une pulsation complexe instantanée pour l'oscillation naturelle $\left(\omega=\omega_{1}+j \omega_{2}\right)$.

Lorsque nous parlerons de pulsations, dans la suite, ce seront des pulsations réelles, au sens courant, que nous envisagerons. Nous préciserons, lorsque ce sera nécessaire, qu'il s'agit de pulsations complexes.

\section{2. Équation différentielle linéaire tangente} et son diagramme de Nyquist. - Il reste à trouver une équation différentielle linéaire dont la loi pseudo-sinusoïdale tangente soit solution. Au voisinage de $t_{0}$, nous pourrons remplacer alors par cette dernière l'équation non linéaire donnée. Une telle équation a déjà été appelée " équation linéaire tangente $)$.

Elle doit être déterminée, dans chaque cas particulier, à partir de l'équation d'oscillation et, peutêtre d'un ordre quelconque. Elle peut donc admettre en plus de la solution pseudo-sinusoïdale qui sert à approcher le phénomène, d'autres solutions. Nous nous placerons toujours dans le cas pratique où ces dernières sont amorties et nous n'en tiendrons pas compte dans l'étude de régimes autoentretenus.

Les coefficients de l'équation tangente dépendent, comme la pseudo-sinusoïde tangente elle-même, du point considéré de la courbe naturelle $a(t)$.

L'étude de Nyquist sera applicable, pour chaque amplitude $a_{t}$, à l'équation linéaire tangente correspondante, ce qui nous permettra d'écrire l'équation aux pulsations complexes sous la forme

$$
H_{i}\left(a_{\ell}, \omega\right)=0 .
$$

On pourra alors construire le diagramme de Nyquist à un paramètre représenté par

$$
H_{i}\left(a_{t}, \omega \text { réel }\right) \text {. }
$$

Ce diagramme dépendant du paramètre $a_{\text {, est }}$ déformable. Il évolue lorsque l'oscillateur passe de l'amorçage à la stabilisation. Tant que l'oscillateur n'a pas atteint un régime d'équilibre, il ne peut passer par l'origine, puisque l'équation $H_{i}\left(a_{t}, \omega\right)=0$ n'admet pas de solution réelle en $\omega$.
Lorsque l'état d'équilibre est atteint, on voit facilement qu'il passe par $O$.

La détermination de l'amplitude d'équilibre revient donc à la recherche d'une valeur du paramètre $\alpha_{t}$ pour laquelle le diagramme de Nyquist passe par $\mathrm{O}$, c'est-à-dire pour laquelle l'équation

$$
H_{i}\left(a_{t},(1)\right)=0
$$

admet une solution réelle en $\omega$.

Si l'on appelle $a_{s}$ et $\omega_{s}$ l'amplitude et la pulsation réelle du régime d'équilibre, on a alors

$$
H_{i}\left(a_{s}, \omega_{s}\right)=0 .
$$

3. Diagramme de Nyquist modifié : $H_{i}\left(a_{t}\right.$, $\omega$ réel) $\times a_{t}$ (multiplication par $a_{t}$ dans le plan complexe). - En étudiant la synchronisation de l'équation de van der Pol (1), nous avons été amené à modifier la représentation d'abord adoptée du diagramme mobile.

Il a été nécessaire de remplacer le diagramme défini à partir du point courant d'affixe $H_{i}\left(a_{t}\right.$, $\omega$ réel) par celui qui est défini à partir du point courant d'affixe $H_{i}\left(a_{\iota}, \omega\right.$ réel $) \times a_{t}$.

Ce dernier se prête mieux à l'étude de certains problèmes, en particulier celui de la détermination de la plage de synchronisation de l'oscillateur. Nous pourrons donc utiliser deux représentations graphiques.: les représentations $H_{i}\left(a_{t}, \omega\right.$ réel $)$ et $H_{i}\left(a_{t}, \omega\right.$ réel $) \times a_{t}$.

4. Courbes équiamplitude et courbes équipulsation. - Le point courant $M$, d'affixe $H_{i}\left(a_{\iota}, \omega_{r}\right) \times a_{\ell} \quad\left(\omega_{r}\right.$ désignant une valeur réelle arbitraire donnée à $\omega$ ), se déduit de l'image $m$ de $H_{i}\left(a_{t}, \omega_{r}\right)$ par l'homothétie de centre $O$ et de rapport $a_{t}$. Si l'on donne à $a_{t}$ une valeur constante, le point $M$ décrit, lorsque $\omega_{r}$ varie, une courbe équiamplitude que nous désignerons par $\left(a_{t}\right)$.

Les courbes obtenues dans les deux représentations sont homothétiques l'une de l'autre par rapport à $\mathrm{O}$.

On peut aussi donner à $\omega$ une valeur réelle constante $\omega_{r}$. Lorsque $a_{t}$ varie, le point M décrit alors une courbe équipulsation que nous désignerons $\operatorname{par}\left(\omega_{r}\right)$.

Chaque point d'une courbe $\left(\omega_{r}\right)$ donnée correspond donc à une valeur différente de $a_{\iota}$. Par suite, les courbes équipulsation obtenues dans les deux représentations ne sont pas homothétiques l'une de l'autre.

On peut préciser l'aspect des courbes $\left(\omega_{r}\right)$ :

Lorsque $a_{t}$ est petit, $H_{i}\left(a_{t}, \omega_{r}\right) \times a_{t}$ peut être remplacé par $H_{i}\left(\omega_{r}\right) \times a_{\ell}$ et l'on voit facilement que les courbes équipulsation correspondantes sont des segments de droites issus de l'origine.

Lorsque $\omega_{r}$ est égal à la pulsation $\omega_{s}$ du régime stabilisé, il existe une valeur de $a_{t}$ pour laquelle $H_{i}\left(a_{t}, \omega_{s}\right)$ est nul : c'est l'amplitude stabilisée $a_{s}$. 
On a alors $H_{i}\left(a_{s}, \omega_{s}\right) \times a_{s}=0$. La courbe équipulsation $\left(\omega_{s}\right)$ passe donc par $\mathrm{O}$, à la fois pour $a_{t}=0$ et pour $a_{t}=a_{s}$; elle a donc une forme de boucle comme le représente la figure $\mathbf{~}$. Cette boucle peut d'ailleurs, dans certains cas particuliers, s'aplatir suivant un segment de droite; un exemple nous en a été donné lorsque nous avons étudié l'équation de van der Pol (1).

Les courbes pour lesquelles $\omega_{r}$ est voisin de $\omega_{\text {; }}$ doivent s'écarter assez peu de la boucle précédente et il est possible de se représenter leur forme de façon très générale (fig. I).

On peut suivre aussi l'évolution d'une courbe équiamplitude pour des valeurs croissantes de $a_{\iota}$, depuis $a_{t}=o$ (on admet que le système est initialement instable). On voit ainsi que cette courbe

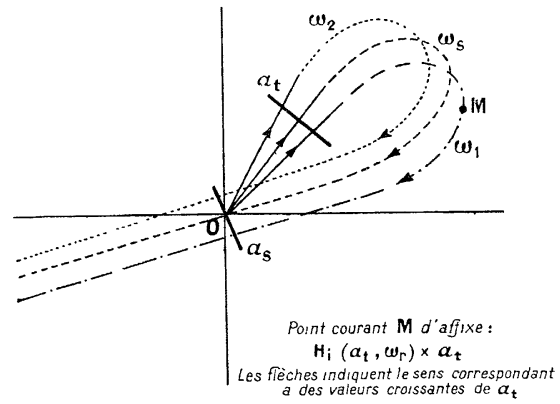

Fig. I.

confondue avec le point $\mathrm{O}$ pour $a_{t}=\mathrm{o}$, passe par $\mathrm{O}$ lorsque $\alpha_{t}$ a atteint la valeur $\alpha_{s}$, c'est-à-dire lorsque l'oscillateur a atteint son régime d'équilibre.

Nous verrons plus loin, en étendant la méthode du diagramme mobile au cas de systèmes à loi linéaire (pour lesquels nous n'avons envisagé jusque-là qu'un diagramme de Nyquist fixe) que, dans ce dernier cas, les courbes équipulsation se réduisent à un faisceau de droites issues de $\mathrm{O}$. Le fait qu'elles ne s'incurvent pas, comme dans les cas non linéaires étudiés, entraîne pour les courbes équiamplitude l'impossibilité de passer par $O$ pour $a_{t} \neq$ o et cela traduit l'impossibilité de stabilisation des oscillateurs à loi linéaire.

5. Trajectoire de l'oscillateur. - Sur chaque courbe équiamplitude $\left(a_{t}\right)$ existe un point particulier correspondant à la pulsation réelle naturelle instantanée. Le lieu de ce point, lorsque $a_{t}$ varie, a été appelé " trajectoire effective de l'oscillateur ".

La trajectoire effective d'un oscillateur n'est généralement pas une courbe équipulsation puisque, comme nous l'avons vu, la pulsation complexe naturelle instantanée varie avec l'amplitude d'oscillation.

Cette trajectoire effective passe évidemment par $\mathrm{O}$ pour $a_{t}=o$. Elle passe à nouveau par O pour $a_{t}=a_{s}$, puisque la pulsation alors réelle et l'amplitude stabilisées vérifient $H_{i}\left(\alpha_{s}, \omega_{s}\right) \times a_{s}=0$.

On peut montrer, de plus, que cette trajectoire effective est, au voisinage de la stabilisation, orthogonale à la famille des courbes équiamplitude correspondantes (appendice II) et qu'il en est de même aux très faibles amplitudes.

Ces remarques apportent donc quelques précisions sur sa forme.

6. Loi fréquence-amplitude. - Au voisinage de la stabilisation, on est amené à assimiler la trajectoire effective à un segment de droite passant par O. La courbe équiamplitude $\left(\omega_{s}\right)$ est aussi assimilable à un segment de droite passant par $O$. La divergence entre ces deux tronçons rectilignes indique donc comment la pulsation naturelle instantanée varie avec l'amplitude au voisinage de stabilisation.

Nous avons exprimé algébriquement cette dépendance pulsation-amplitude dans certains cas particuliers. Il est possible de donner maintenant des formules plus générales exprimant cette loi.

On peut montrer, en particulier (appendice I), que, au voisinage de la stabilisation, les variations $\delta \omega_{1}$ et $\delta \omega_{2}$ apportées à la partie réelle et imaginaire pure de la pulsation complexe par une variation d'amplitude $\delta a$ sont données par

$$
\begin{aligned}
& {\left[\frac{\partial U}{\partial \omega} \frac{\partial U}{\partial a}+\frac{\partial V}{\partial \omega} \frac{\partial V}{\partial a}\right]_{a=a_{s}}} \\
& \delta \omega_{1}=-\frac{\omega_{\omega}=\omega_{s}}{\left(\frac{\partial U}{\partial \omega}\right)^{2}+\left(\frac{\partial V}{\partial \omega}\right)^{2}} \delta, \\
& \delta \omega_{2}=\frac{\left[\frac{\partial U}{\partial a} \frac{\partial V}{\partial \omega}-\frac{\partial U}{\partial \omega} \frac{\partial V}{\partial a}\right]_{\substack{a=a_{s} \\
\omega=\omega_{s}}} \delta a ;}{\left(\frac{\partial U}{\partial \omega}\right)^{2}+\left(\frac{\partial V}{\partial \omega}\right)^{2}}
\end{aligned}
$$

$U$ et $V$ sont les parties réelle et imaginaire de $H_{i}\left(a_{t}, \omega_{r}\right) ; \omega_{s}$ est toujours la pulsation du régime stabilisé.

$\omega_{s}+\delta \omega_{1}$ est alors la pulsation réelle instantanée de l'oscillation naturelle quand son amplitude est $a_{t_{0}}=a_{s}+\delta a$, la pulsation complexe instantanée correspondante étant $\omega_{s}+\delta \omega_{1}+j \delta \omega_{2}$.

L'expression $\delta \omega_{2}$ est évidemment liée aux constantes de temps $\tau_{1}$ et $\tau_{2}$ introduites antérieurement, les expressions de ces constantes de temps dans le cas général seront données dans l'appendice I.

L'importance de la dépendance pulsation-amplitude est capitale.

On voit, en particulier, que toute modulation d'amplitude doit entraîner une modulation de fréquence.

La modulation d'amplitude peut être produite par des chocs : chocs mécaniques ou chocs électroniques tels que ceux qui prennent naissance dans les résistances, par suite de l'agitation thermique. 
Il en résulte donc une instabilité de la fréquence qui limite, par exemple, l'emploi de l'oscillateur comme horloge radioélectrique.

\section{Limites de la plage de synchronisation.} - Nous reprendrons l'équation différentielle non linéaire d'oscillation en $\mathrm{y}$ ajoutant un second membre sinusoïdal $E_{0} \sin \left(\omega_{0} t+\varphi\right)$. On peut se ramener comme plus haut à l'étude d'une équation linéaire tangente dont les coefficients dépendent du paramètre $a_{t}$ et dont le second membre est le terme sinusoïdal donné. Le remplacement de la variable $V$ inconnue et $\mathrm{du}$ second membre, respectivement par $a_{0} \mathrm{e}^{j \psi} \mathrm{e}^{j \omega_{0} t}$ et $E_{0} \mathrm{e}^{j o} \mathrm{e}^{j \omega_{0} l}$, nous donne encore une équation de la forme

$$
H_{i}\left(a_{0}, \omega_{0}\right) a_{0} \mathrm{e}^{j \psi}=\boldsymbol{E}_{0} \mathrm{e}^{j \varphi}
$$

qui permettra la détermination de $a_{0}$ (amplitude de la solution synchronisée) et de $\psi(\psi-\varphi$ étant le

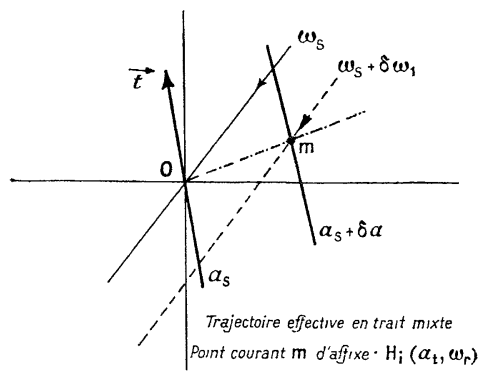

Fig. 2 .

déphasage entre la solution synchronisée et le terme d'attaque).

La résolution graphique, développée sur un exemple, est applicable dans le cas général où elle est aussi facilement justifiée.

7. x. Construction. - Nous supposons tracé [en adoptant la représentation $H_{i}\left(a_{t}, \omega_{r}\right) \times a_{t}$ ] un réseau aussi dense que possible de courbes équiamplitude et de courbes équipulsation.

Soit alors $\mathrm{M}_{0}$ le point du plan représentant le nombre complexe $E_{0} \mathrm{e}^{j}$. . Le cercle de centre $\mathrm{O}$ et de rayon $\mathrm{OM}_{0}=E_{0}$ coupe la courbe équipulsation $\left(\omega_{0}\right)$ au point $M(f i g .3)$.

$I^{\circ}$ L'angle des rayons vecteurs $\mathrm{OM}$ et $\mathrm{OM}_{0}$ a pour valeur $\psi$.

$2^{0}$ La courbe équiamplitude passant par $\mathrm{M}$ définit la valeur de l'amplitude de la solution synchronisée.

7.2. Discussion de L'intersection. - Il résulte de la forme des courbes équipulsation étudiée plus haut, que le cercle de centre $O$, de rayon $E_{0}$, pourra couper la courbe $\left(\omega_{0}\right)$ en plus d'un point.

En particulier, si le rayon $E_{0}$ du cercle est petit et si $\omega_{0}$ est assez voisin de $\omega_{s}$, il y aura au moins trois points d'intersection comme le montre la figure 3 .
Ces points ne peuvent cependant convenir que s'ils correspondent à des solutions stables et nous voyons qu'il sera nécessaire de joindre à la discussion

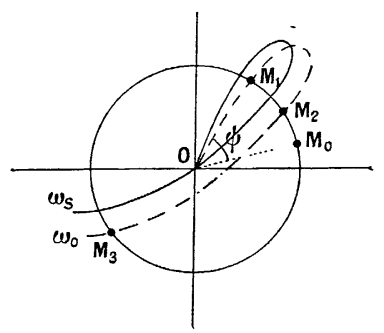

Fig. 3 .

géométrique une autre discussion précisant la stabilité ou l'instabilité des solutions trouvées.

Si nous diminuons l'amplitude $E_{0}$ de l'attaque, la pulsation d'attaque restant fixe, nous voyons que le nombre de solutions peut, néanmoins, être inférieur à trois.

Une limite apparaît lorsque le cercle de rayon $E_{\mathbf{0}}$ est tangent à la courbe $\left(\omega_{0}\right)$ considérée. Dans ce cas, deux des solutions viennent se confondre.

Si le rayon $E_{0}$ est inférieur à ce rayon limite, il ne reste plus qu'une solution et, comme nous verrons qu'elle est certainement instable, la synchronisation n'est alors plus possible.

\subsection{Limites DE LA PLAGE DE Synchronisation.} - Le contact entre le cercle de rayon $E_{0}$ et la courbe $\left(\omega_{0}\right)$ fait donc apparaître une limite de synchronisation (à supposer que les solutions qui peuvent ainsi disparaître soient stables auparavant).

Il est possible de déterminer le lieu de tels points de contact quand $\omega_{0}$ varie (courbe limite $\boldsymbol{\Gamma}$ en trait mixte de la figure 4) et d'en déduire la largeur de la plage de synchronisation.

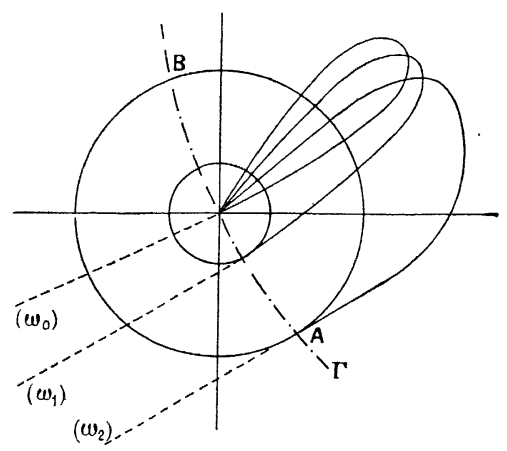

Fig. 4 .

Cette plage sera définie, pour une amplitude d'attaque $E_{0}$ donnée, par l'ensemble des valeurs que peut prendre $\omega_{0}$ pour qu'il y ait synchronisation. Elle est représentée sur le dessin par la portion de courbe $\boldsymbol{\Gamma}$ intérieure au cercle de rayon $E_{0}$. 
Les valeurs de la pulsation d'attaque au delà desquelles la synchronisation n'est plus possible sont évidemment données par les deux courbes équipulsation limites tangentes au cercle de rayon $E_{0}$

7.4. Stabilité de La synchronisation. On suppose que la solution trouvée subit une petite modification $\Delta V$ de module $\xi$ et l'on étudie l'évolution ultérieure de $\xi$. Si $\xi$ tend vers zéro, la solution est stable; si $\zeta$ croît indéfiniment, la solution est instable.

L'étude assez compliquée dans le cas général prend une forme simple lorsque le paramètre $a_{t}$, qui figure dans $H_{i}\left(a_{t}, \omega_{r}\right)$, n'intervient que par son carré $a_{t}^{2}$. Il en est ainsi (1) lorsque l'équation non linéaire d'oscillation peut être mise sous la forme d'un développement tel que

$$
\left(H_{1}\right)_{0 p} V+\left(H_{3}\right)_{o p} V^{3}=o
$$

ce qui est le cas pour un très grand nombre d'oscillateurs.

On peut alors former une équation différentielle du second ordre décrivant l'évolution de $\xi$ de la façon suivante qui sera justifiée dans l'appendice III.

${ }^{\circ}{ }^{\circ}$ On remplace $H_{i}\left(a_{0}, j \omega_{0}\right)$ par $H_{i}\left(a_{0}, j \omega_{0}+q\right)$, $q$ étant l'opérateur $\frac{\mathrm{d}}{\mathrm{d} t}\left(a_{0}\right.$ et $\omega_{0}$ étant toujours l'amplitude et la pulsation de l'oscillation synchronisée). $2^{\circ}$ On néglige dans l'expression précédente les termes du second ordre en $q$ et au delà (ce qui est justifié si $\xi$ est lentemènt variable).

$3^{\circ}$ L'équation cherchée, du second ordre à coefficients constants est

$$
\left[\mathrm{I}+a^{2} \frac{\partial}{\partial\left(a^{2}\right)}\right]_{a=a_{0}}\left|\boldsymbol{H}\left(a_{0}, j \omega_{0}, q\right)\right|^{2} \xi=0 ;
$$

une application de cette formule est donnée dans l'appendice III.

8. Continuité des propriétés physiques d'un auto-oscillateur dans le passage de ses états quasi linéaires aux régimes nettement non linéaires. - Nous montrerons, pour conclure cette étude des auto-oscillateurs, qu'il est possible, lorsque l'oscillateur est régi par une loi linéaire, de lui faire correspondre aussi un diagramme de Nyquist mobile.

Il suffit, pour cela, de remplacer, comme nous l'avons fait dans le cas où la loi est non linéaire, le point courant $m$ image de $H_{i}\left(\omega_{r}\right)$ par le point courant M image de $H_{i}\left(\omega_{r}\right) \times a_{t}\left(a_{t}\right.$ désignant toujours l'amplitude d'oscillation à un instant donné).

8. I. Courbes ÉQUiAmplitude ET COURBES ÉQUiPULSATION. - Le réseau des courbes équiamplitude

(1) Les oscillateurs non linéaires et le diagramme de Nyquist. et des courbes équipulsation sera construit facilement à partir de la courbe fixe de Nyquist représentée par $H_{i}$ ( $\omega$ réel).

Les courbes équiamplitude se déduisent de cette dernière par l'homothétie de centre $O$ et de rapport $a_{t}, a_{t}$ ayant pour chaque courbe une valeur donnée.

Les courbes équipulsation sont obtenues en donnant à $\omega$ une valeur réelle constante $\omega_{r}$. Chaque point d'une courbe équipulsation est donc déduite d'un même point fixe de la courbe fixe de Nyquist. Ces courbes se réduisent donc ici à un faisceau de demidroites issues de 0 .

Les courbes équiamplitude et équipulsation seront encore désignées par $\left(a_{t}\right)$ et $\left(\omega_{r}\right)$.

On peut suivre, comme dans le cas des oscillateurs non linéaires, l'évolution du diagramme de

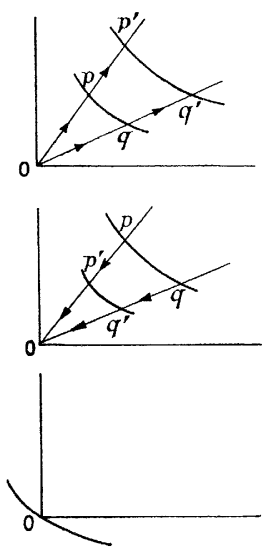

Fig. 5.

Nyquist à un paramètre que nous venons de définir (fig. 5).

L'amplitude d'oscillation $a_{t}$ étant supposée initialement différente de zéro, l'oscillateur peut se comporter de diverses façons; supposons d'abord que la condition d'accrochage ne soit pas exactement vérifiée :

$1^{\circ}$ Le système peut être instable. La courbe de Nyquist mobile ne passe pas initialement par l'origine et tous ses points s'éloignent ensuite de $O$ suivant la loi d'évolution indiquée plus haut (homothétie de rapport croissant).

$2^{\circ}$ Le système peut tendre vers le repos. La courbe ne passe pas initialement par l'origine et tous ses points s'en rapprochent, se confondant à la limite en $\mathrm{O}$.

Si la condition d'accrochage est exactement vérifiée, la courbe passe par $O$ et le système n'évolue pas (état d'équilibre indifférent).

On voit qu'un régime d'oscillation stable ne peut jamais s'établir et que cela tient à l'impossibilité, pour la courbe mobile, de passer par l'origine en 
dehors du cas limite où la condition d'accrochage est vérifiée.

Ce passage par l'origine, pour une valeur convenable de l'amplitude, est, au contraire, permis à la courbe de Nyquist mobile d'un système non linéaire.

8.2. Trajectoire de L'oscillateur. - Sur chaque courbe équiamplitude $\left(a_{t}\right)$ se trouve un point $\mathrm{M}_{0}$ particulier correspondant à la partie réelle de la pulsation complexe naturelle. Le lieu de ce point lorsque $\left(a_{t}\right)$ varie est la « trajectoire effective de l'oscillateur " précédemment définie.

Puisque la pulsation complexe naturelle est, pour un système linéaire, indépendant de l'amplitude, la trajectoire effective d'un tel oscillateur est une courbe équipulsation particulière. C'est donc une demi-droite issue de $\mathrm{O}$.

On peut montrer aisément que cette demi-droite est orthogonale à la famille des courbes $\left(a_{t}\right)$. Cela tient au fait que, sur chaque courbe $\left(a_{t}\right)$ le point $\mathrm{M}_{0}$ correspond à un extremum du rayon vecteur.

8.3. Oscillation fORGÉE. - Nous reprendrons encore l'équation d'oscillation en y ajoutant un second membre sinusoïdal donné, elle devient

$$
H_{\mathrm{op}} V=E_{0} \sin \left(\omega_{0} t+\varphi\right) .
$$

Cette équation peut être résolue par la méthode graphique indiquée précédemment pour un oscillateur non linéaire. Nous nous contenterons de rappeler ici la construction utilisée.

Nous prenons sur le plan complexe le point $\mathrm{M}_{0}$ d'affixe $E_{0} \mathrm{e} j \varphi$, nous traçons un cercle de centre O, de rayon $E_{0}$ qui coupe la demi-droite $\left(\omega_{0}\right)$ au point M (fig. 6).

$1^{\circ}$ L'angle des rayons vecteurs $\mathrm{OM}$ et $\mathrm{OM}_{0}$ a pour valeur $\psi$.

$2^{\circ}$ La courbe équiamplitude $\left(a_{0}\right)$ passant par $\mathrm{M}$ définit la valeur de l'amplitude de la solution.

$3^{\circ}$ La solution cherchée est $a_{0} \sin \left(\omega_{0} t+\psi\right)$.

La construction, toujours possible, admet une solution unique puisque nous supposons que la demi-droite $\left(\omega_{0}\right)$ est unique. Cette solution ne pourra convenir cependant que si elle est stable.

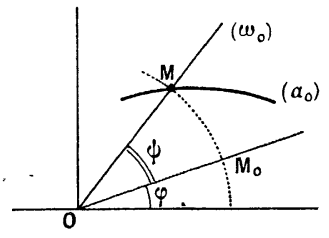

Fig. 6.

Stabilité de la solution. - On fera subir, là encore, une petite modification $\Delta V$ de module $\xi$ à la solution trouvée et l'on étudiera l'évolution ultérieure de $\zeta^{\circ}$.
Le raisonnement est simplifié par le fait que $H_{\mathrm{op}}$ est ici un opérateur linéaire. On a, en effet,

$$
H_{\mathrm{op}}(V+\Delta V)=H_{\mathrm{op}} V+H_{\mathrm{op}} \Delta V=E_{0} \sin \left(\omega_{0} t+\varphi\right)
$$

ou, tenant compte de l'équation (5),

$$
H_{\text {op }} \Delta V=\text { o. }
$$

Cette dernière équation nous montre que si l'oscillation libre du système est instable, la solution forcée est aussi instable. On ne peut donc définir une oscillation forcée stable que pour des systèmes dont l'oscillation libre est amortie.

Cette méthode fait apparaître l'analogie entre la solution forcée d'un oscillateur régi par une loi linéaire et la solution synchronisée d'un oscillateur régi.par une loi non linéaire.

Dans le cas d'un oscillateur non linéaire, les courbes équipulsation $\left(\omega_{r}\right)$ issues toutes de $\mathrm{O}$ s'incurvent lorsque l'on s'éloigne de l'origine. Au voisinage de $\mathrm{O}$, on peut, néanmoins assimiler ce faisceau de courbes à un faisceau de segments de droites et, par suite, approcher l'équation non linéaire donnée par une équation linéaire.

Cette équation est évidemment une équation linéaire tangente particulière correspondant aux faibles amplitudes d'oscillation.

En étudiant la synchronisation des systèmes non linéaires, nous avons obtenu une solution correspondant à cette portion linéaire du faisceau de courbes $\left(\omega_{r}\right)$; elle ne peut donc être stable, puisque le système est supposé autoentretenu.

\section{APPENDIGE I.}

Les parties réelle et imaginaire pure de $H_{i}\left(a_{t}, \omega_{r}\right)$ seront mises en évidence en écrivant cette expression sous la forme

$$
\boldsymbol{H}_{\boldsymbol{i}}\left(a_{t}, \omega_{r}\right) \equiv \boldsymbol{U}\left(a_{t}, \omega_{r}\right)+j \boldsymbol{V}\left(a_{t}, \omega_{r}\right) ;
$$

$U\left(a_{t}, \omega_{r}\right)$ et $V\left(a_{t}, \omega_{r}\right)$ sont des expressions réelles qui représentent les coordonnées du point $m$, considéré précédemment, dont l'affixe est $H_{i}\left(a_{t}, \omega_{r}\right)$ (fig. 2).

Nous supposerons que, à l'instant où nous considérons le système, son régime est stabilisé; $m$ est alors en $\mathrm{O}$ comme nous l'avons vu.

Considérons un état voisin pour lequel la stabilisation n'est pas atteinte. Le point $m$ représentant cet état se trouve sur la portion de trajectoire effective [dans la représentation $H_{i}\left(a_{t}, \omega_{r}\right)$ ] voisine de O. L'amplitude a pour valeur $a_{t}=a_{s}+\delta a$ et la pulsation complexe

\section{L'équation}

$$
\omega=\omega_{s}+\delta \omega_{1}+j \delta\left(\omega_{2} .\right.
$$

$$
H_{i}\left(a_{t}, \omega\right)=\mathrm{o},
$$

qui relie la pulsation complexe instantanée de l'oscil- 
lation naturelle à son amplitude $a_{t}$ prend la forme

$$
\begin{aligned}
& \boldsymbol{H}_{i}\left(a_{s}+\delta a, \omega_{s}+\delta \omega_{1}+j \delta \omega_{2}\right) \\
& \equiv U\left(a_{s}, \omega_{s}\right)+j V\left(a_{s}, \omega_{s}\right)+\delta a\left[\frac{\partial U}{\partial a}+j \frac{\partial V}{\partial a}\right]_{\substack{a=a_{s} \\
\omega=\omega_{s}}} \\
& +\left(\delta \omega_{1}+j \delta \omega_{2}\right)\left[\frac{\partial U}{\partial \omega}+j \frac{\partial V}{\partial \omega} \cdot\right]_{\substack{a=a_{s} \\
\omega=\omega_{s}}}=0
\end{aligned}
$$

ou, puisque $\operatorname{Ii}\left(a_{s}, \omega_{r}\right)=V\left(a_{s}, \omega_{r}\right)=\mathrm{o}$,

$$
\begin{aligned}
& \delta a\left[\frac{\partial U}{\partial a}+j \frac{\partial V}{\partial a}\right]_{\substack{a=a_{s} \\
\omega=\omega_{s}}} \\
& +\left(\delta \omega_{1}+j \delta \omega_{2}\right)\left[\frac{\partial U}{\partial \omega}+j \frac{\partial V}{\partial \omega}\right]_{\substack{a=a_{s} \\
\omega=\omega_{s}}}=0 .
\end{aligned}
$$

En égalant à zéro les parties réelle et imaginaire pure du premier membre, on obtient $\left(^{2}\right)$

$$
\begin{aligned}
& {\left[\frac{\partial U}{\partial \omega} \frac{\partial U}{\partial a}+\frac{\partial V}{\partial \omega} \frac{\partial V}{\partial \alpha}\right]_{a=a_{s}}}
\end{aligned}
$$

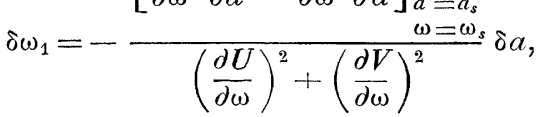

$$
\begin{aligned}
& \delta \omega_{2}=\frac{\left[\frac{\partial U}{\partial a} \frac{\partial V}{\partial \omega}-\frac{\partial U}{\partial \omega} \frac{\partial V}{\partial a}\right]_{\substack{a=a_{s} \\
\omega=\omega_{s}}} \delta a .}{\left(\frac{\partial U}{\partial \omega}\right)^{2}+\left(\frac{\partial V}{\partial \omega}\right)^{2}}
\end{aligned}
$$

Au voisinage de la stabilisation la pseudo-sinusoïde tangente est donc de la forme

$$
V=a_{t_{0}} \mathrm{e}^{-\hat{\partial} \omega_{2}\left(\iota-t_{0}\right)} \mathrm{e}^{j\left(\omega_{s}+\hat{\partial} \omega_{1}\right) t} .
$$

Remarquons que $\delta \omega_{1}$ et $\delta \omega_{2}$ peuvent être mis sous la forme

$$
\begin{aligned}
& \delta \omega_{1}=-\frac{\left|\begin{array}{ll}
\frac{\partial U}{\partial \omega} & -\frac{\partial V}{\delta \omega} \\
\frac{\partial V}{\partial a} & \frac{\partial U}{\partial a}
\end{array}\right|}{\left|\begin{array}{ll}
\frac{\partial U}{\partial \omega} & -\frac{\partial V}{\partial \omega} \\
\frac{\partial V}{\partial \omega} & \frac{\partial U}{\partial \omega}
\end{array}\right|} \delta a, \\
& \delta \omega_{2}=\frac{\left|\begin{array}{ll}
\frac{\partial U}{\partial a} & \frac{\partial U}{\partial \omega} \\
\frac{\partial V}{\partial a} & \frac{\partial V}{\partial \omega}
\end{array}\right|}{\left|\begin{array}{ll}
\frac{\partial U}{\partial \omega} & -\frac{\partial V}{\partial \omega} \\
\frac{\partial V}{\partial \omega} & \frac{\partial U}{\partial \omega}
\end{array}\right|} \delta a .
\end{aligned}
$$

Constantes de temps $\tau_{1}$ et $\tau_{2}$. - La définition de ces constantes de temps a été donnée dans l'exposé précédent ${ }^{(1)}$.

(2) Cette démonstration est due à J. Loes, Ann. des Télécom., déc. 195 r, qui a poursuivi sur les servomécanismes des recherches parallèles aux nôtres (A. Blaquières, C. R. Acad. Sc., 1 95 г, 233, 345; 1951, 233, 1 $434 ;$ 1 952,234, 1 74 1).
La constante de temps instantanée de l'oscillation naturelle est

$$
\tau_{1}=\frac{\mathrm{I}}{\delta \omega_{2}} .
$$

La constante de temps $\tau_{2}$ qui caractérise le retour à l'équilibre de l'oscillateur, supposé légèrement écarté de cet état, sera obtenue suivant la méthode déjà développée sur un exemple.

On exprimera d'abord le contact, à l'instant $t_{0}$, entre la pseudo-sinusoïde tangente et la courbe $a(t)$ représentant l'évolution de l'amplitude de l'oscillation naturelle.

On a donc

$$
\frac{\mathrm{I}}{a_{l_{0}}}\left(\frac{\mathrm{d} u}{\mathrm{~d} t}\right)_{l_{0}}=-\delta \omega_{2}
$$

ou

en posant

$$
\left(\frac{\mathrm{d} a}{\mathrm{~d} t}\right)_{\iota_{0}}+\frac{|N|}{|D|} a_{t_{0}} \delta a=0
$$

$$
\begin{aligned}
& |N|=\left|\begin{array}{ll}
\frac{\partial U}{\partial a} & \frac{\partial U}{\partial \omega} \\
\frac{\partial V}{\partial a} & \frac{\partial V}{\partial \omega}
\end{array}\right|, \\
& |D|=\left|\begin{array}{ll}
\frac{\partial U}{\partial \omega} & -\frac{\partial V}{\partial \omega} \\
\frac{\partial V}{\partial \omega} & \frac{\partial U}{\partial \omega}
\end{array}\right|,
\end{aligned}
$$

Il est commode de prendre alors ò comme nouvelle variable. (Nous poserons, pour simplifier l'écriture, $a=\varepsilon$, avec $\varepsilon=a_{t_{0}}-a_{s}$.)

Compte tenu de ce que $\varepsilon$ est petit, l'équation ci-dessus peut être remplacée par

$$
\left(\frac{\mathrm{d} \varepsilon}{\mathrm{d} t}\right)_{t_{0}}+\frac{|N|}{|D|} a_{s} \varepsilon=0 .
$$

Cette équation, valable quel que soit $t_{0}$, donne la loi d'évolution de $\varepsilon$ et traduit le 'retour à l'équilibre de l'oscillateur. On en tire

$$
\varepsilon=\mathrm{e}^{-\frac{|N|}{|D|} a_{s} t} .
$$

D'où l'expression de $\tau_{2}$

$$
\tau_{2}=\frac{I}{a_{s}} \frac{\left|\begin{array}{ll}
\frac{\partial U}{\partial \omega} & -\frac{\partial V}{\partial \omega} \\
\frac{\partial V}{\partial \omega} & \frac{\partial U}{\partial \omega}
\end{array}\right|}{\left|\begin{array}{ll}
\frac{\partial U}{\partial a} & \frac{\partial U}{\partial \omega} \\
\frac{\partial V}{\partial a} & \frac{\partial V}{\partial \omega}
\end{array}\right|} .
$$

\section{APPENDIGE II.}

On a représenté, sur la figure 2, la courbe $H\left(a_{s}+\delta a, \omega\right.$ réel) voisine de la courbe équiamplitude $\left(a_{s}\right)$ correspondant à la stabilisation. 
Le point $m$ de cette courbe correspondant aux valeurs effectives

$$
a_{t}=a_{s}+\delta a, \quad \text { (1) réel }=\left(\omega_{s}+\delta()_{1},\right.
$$

a pour coordonnées

$$
\left(\frac{\partial U}{\partial a}\right)_{\substack{a_{s} \\ \omega_{s}}} \delta a+\left(\frac{\partial U}{\partial \omega}\right)_{\substack{a_{s} \\ \omega_{s}}} \delta \omega_{1}
$$

et

$$
\left(\frac{\partial V}{\partial a}\right)_{\substack{a_{s} \\ \omega_{s}}} \delta a+\left(\frac{\delta V}{\delta \omega}\right)_{\substack{a_{s} \\ \omega_{s}}} \delta\left(\omega_{1}\right.
$$

Le vecteur $\vec{t}$ de composantes $\left(\frac{\partial U}{\partial \omega}\right)_{\substack{a_{s} \\ \omega_{s}}}$ et $\left(\frac{\partial V}{\partial \omega}\right)_{a_{s}}$ étant tangent à la courbe $\left(a_{s}\right)$ en $\mathrm{O}$, nous connaîtrons l'angle de $\overrightarrow{\mathrm{Om}}$ et de cette courbe en formant le produit scalaire $\vec{t} \cdot \overrightarrow{\mathrm{Om}}$. Son expression est

$$
\begin{aligned}
\vec{t} \cdot \overrightarrow{O m}= & \left(\frac{\partial U}{\partial \omega} \frac{\partial U}{\partial a}+\frac{\partial V}{\partial \omega} \frac{\partial V}{\partial a}\right)_{\substack{a_{s} \\
\omega_{s}}} \hat{} \\
& +\left[\left(\frac{\partial L^{T}}{\partial(\omega)}\right)^{2}+\left(\frac{\partial V}{\partial(1)}\right)^{2}\right]_{\substack{a_{s} \\
\omega_{s}}} \delta \omega_{1}
\end{aligned}
$$

il est nul comme le montre l'égalité (6) et, par suite, $\overrightarrow{\mathrm{Om}}$ est normal à $\vec{t}$.

Cette propriété, démontrée pour les courbes du diagramme $H_{i}\left(a_{t}, \omega\right.$ réel), est aussi valable pour les courbes $H_{i}\left(a_{t}, \omega\right.$ réel $) \times a_{t}$ comme on s'en rend compte aisément en faisant subir à ces dernières l'homothétie de centre $\mathrm{O}$ et de rapport $a_{t}$.

La condition pour que la pulsation de l'oscillation naturelle soit indépendante de l'amplitude est, par suite,

$$
\frac{\partial U}{\partial \omega} \frac{\partial U}{\partial a}+\frac{\partial V}{\partial \omega} \frac{\partial V}{\partial a}=0 ;
$$

elle exprime l'orthogonalité du tronçon rectiligne de courbe $\left(\omega_{s}\right)$ et du vecteur $\vec{t}$. La trajectoire effective se confond alors avec la courbe équipulsation $\left(\omega_{s}\right)$ passant par $\mathrm{O}$, au voisinage de $\mathrm{O}$.

\section{APPENDIGE III.}

Revenons à l'équation qui nous a servi à étudier la synchronisation d'un oscillateur non linéaire et que l'on peut écrire sous la forme

$H_{\mathrm{op}}\left(a_{t}, \frac{\mathrm{d}}{\mathrm{d} t}, \frac{\mathrm{d}^{2}}{\mathrm{~d} t^{2}}, \ldots\right) a_{t} \sin \left(\omega_{0} t+\psi\right)=E_{0} \sin \left(\omega_{0} t+\xi\right)$.

Nous avons cherché une solution synchronisée d'amplitude constante $a_{0}$ et nous avons vu que pour déterminer cette amplitude $a_{0}$ il suffit de remplacer $\left.\left(j \omega_{0}\right)^{2}, \ldots\right]$.
Pour savoir si la solution trouvée est stable, nous devons étudier le comportement du système lorsqu'on le suppose dans un état voisin du régime synchronisé envisagé. Il faut alors tenir compte des variations de l'amplitude d'oscillation $a_{t}$ avec le temps et prévoir si $a_{t}$ tend vers la valeur $a_{0}$ ou s'en écarte.

On pourrait étudier l'évolution de l'oscillateur au voisinage d'un état synchronisé en introduisant, comme nous l'avons fait jusque-là, une pulsation complexe. Nous utiliserons cependant ici pour plus de clarté une autre forme d'exposé en n'envisageant que des fonctions dont la pulsation est réelle et l'amplitude variable avec le temps. Les deux méthodes se ramènent d'ailleurs immédiatement l'une à l'autre.

Le formalisme du calcul symbolique est aisément applicable aux fonctions considérées si l'on remarque que les dérivées

$$
\begin{aligned}
\frac{\mathrm{d}}{\mathrm{d} t}\left(a_{\iota} \sin \omega t\right)= & a_{t} \frac{\mathrm{d}}{\mathrm{d} t}(\sin \omega t)+\sin \omega t \frac{\mathrm{d} a}{\mathrm{~d} t}, \\
\frac{\mathrm{d}^{2}}{\mathrm{~d} t^{2}}\left(a_{t} \sin \omega t\right)= & a_{\iota} \frac{\mathrm{d}^{2}}{\mathrm{~d} t^{2}}(\sin \omega t) \\
& +2 \frac{\mathrm{d} a}{\mathrm{~d} t} \frac{\mathrm{d}}{\mathrm{d} t}(\sin \omega \iota)+\frac{\mathrm{d}^{2} a}{\mathrm{~d} t^{2}} \sin \omega t
\end{aligned}
$$

peuvent être écrites sous la forme réduite

$$
\begin{aligned}
\frac{\mathrm{d}}{\mathrm{d} t}\left(a_{t} \sin \omega t\right) & =(p+q) a \sin \omega t \\
\frac{\mathrm{d}^{2}}{\mathrm{~d} t^{2}}\left(a_{\iota} \sin \omega t\right) & =(p+q)^{2} a \sin \omega t
\end{aligned}
$$

en posant

$$
p^{m}=\left(\frac{\partial^{m}}{\partial t^{m}}\right)_{a}, \quad q^{n}=\left(\frac{\partial^{n}}{\partial t^{n}}\right)_{\sin \omega \iota} .
$$

Plus généralement, on sait que si deux fonctions $u$ et $v$ dépendent du temps, on a

$$
\frac{\mathrm{d}^{m}}{\mathrm{~d} t^{m}}(u v)=(p+q)^{m} u v,
$$

avec

$$
p^{m}=\left(\frac{\partial^{m}}{\partial t^{m}}\right)_{u}, \quad q^{n}=\left(\frac{\partial^{n}}{\partial t^{n}}\right)_{,},
$$

Il en résulte que si l'on veut tenir compte à la fois des variations de $a_{\iota}$ et de $\sin \left(\omega_{0} t+\psi\right)$, on sera amené à remplacer l'opérateur qui figure dans l'équation d'oscillation par le nouvel opérateur

$$
H_{\mathrm{op}}\left[a_{\iota},(p+q),(p+q)^{2}, \ldots\right] \text {. }
$$

Si $a_{t}$ devient constant, on a $q=0$ et l'opérateur $H_{\mathrm{op}}$ se réduit à celui que nous avons utilisé pour trouver les solutions synchronisées : $H_{\mathrm{op}}\left(a_{t}\right.$, $\left.p, p^{2}, \ldots\right)$.

Si $a_{t}$ est lentement variable à côté de $\sin \omega_{0} t$, on pourra, dans les calculs, considérer $q$ comme un paramètre petit devant $p$ et utiliser les méthodes d'approximation classiques du calcul algébrique.

On voit facilement que l'on peut remplacer encore 
les expressions réelles $a_{t} \sin \left(\omega_{0} t+\psi\right)$ et $E_{0} \sin \left(\omega_{0} t+\varphi\right)$ qui figurent dans l'équation

$$
\begin{aligned}
& \boldsymbol{H}_{\mathrm{op}}\left[a_{t},(p+q),(p+q)^{2}, \ldots\right] a_{t} \sin \left(\omega_{0} t+\psi\right) \\
& \quad=E_{0} \sin \left(\omega_{0} t+\varphi\right)
\end{aligned}
$$

par les expressions complexes

$$
V=a_{t} \mathrm{e}^{j\left(\omega_{0} t+\psi\right)}, \quad E=E_{0} \mathrm{e}^{j\left(\omega_{0} t+\varphi\right)}
$$

(avec $\omega_{0}$ réel et $a_{t}$ réel, variable avec le temps). Si nous supposons, de plus, comme nous en avons fait antérieurement l'hypothèse, que $a_{t}$ figure au second degré dans la relation précédente, on pourra écrire cette dernière sous la forme

$$
\boldsymbol{H}_{\mathrm{op}}\left[a_{\ell}^{2},\left(j \omega_{0}+q\right),\left(j \omega_{0}+q\right)^{2}, \ldots\right] \boldsymbol{V}=E
$$

ou, plus simplement,

$$
H_{\mathrm{op}}\left[a_{t}^{2}, j \omega_{0}+q\right] V=E .
$$

Faisons alors subir à $V$, à partir de l'état

$$
V_{0}=a_{0} \mathrm{e}^{i\left(\omega_{0} t+\grave{\varphi}\right)}
$$

correspondant à une solution synchronisée, une petite modification $\Delta V$ de module $\xi$. Il vient

$$
H\left[a_{0}^{2}+\Delta\left(a_{t}^{2}\right), j \omega_{0}+q\right]\left(V_{0}+\Delta V\right)=E
$$

ou au premier ordre

$$
\begin{gathered}
H\left(a_{0}^{2}, j \omega_{0}\right) V_{0}+H\left(a_{0}^{2}, j \omega_{0}+q\right) \Delta V \\
\quad+\left[\frac{\partial H}{\partial\left(a_{l}^{2}\right)}\right]_{a_{t}=a_{0}} V_{0} \Delta\left(a_{t}^{2}\right)=E .
\end{gathered}
$$

Tenant compte de l'égalité $H\left(a_{0}^{2}, j \omega_{0}\right) V_{0}=E$, on en déduit

$$
H\left(a_{0}^{2}, j \omega_{0}+q\right) \Delta V+\frac{\partial H}{\partial\left(a_{t}^{2}\right)} V_{0} \Delta\left(a_{t}^{2}\right)=0 .
$$

Cette expression peut être mise sous une forme plus utilisable si l'on remarque que l'opérateur $q$ peut être considéré, pour la commodité des calculs, comme un paramètre réel (cela tient au fait qu'il n'opère que sur $\xi$ qui est une fonction réelle). On peut alors exprimer que le premier membre de l'égalité précédente a un module nul, ce qui donne

$$
\left|\boldsymbol{H}\left(a_{0}^{2}, \omega_{0}, q\right)\right| \xi+2\left[\frac{\left|\partial \boldsymbol{H}\left(a_{t}^{2}, \omega_{0}, q\right)\right|}{\partial\left(a_{t}^{2}\right)}\right]_{a_{t}=a_{0}} a_{0}^{2} \xi=0
$$

ou, en multipliant les deux membres par $\mid H\left(a_{0}, \omega_{0}, q\right)$,

$$
\left[\mathrm{I}+a_{t}^{2} \frac{\partial}{\partial\left(a_{t}^{2}\right)}\right]_{a_{t}=a_{0}}\left|\boldsymbol{H}\left(a_{t}, \omega_{0}, q\right)\right|^{2} \xi=\mathbf{o} .
$$

On négligera, dans l'expression $\left|H\left(a_{t}, \omega_{0}, q\right)\right|^{2}$, les termes en $a$ de degré supérieur à deux.

L'équation ainsi trouvée, qui est une équation différentielle du second ordre en $\xi$, si l'on remplace, à la fin des calculs, $q$ par l'opérateur $\frac{\mathrm{d}}{\mathrm{d} t}$ et $q^{2}$ par $\frac{\mathrm{d}^{2}}{\mathrm{~d} t^{2}}$, permet d'étudier l'évolution de $\xi$.
Application à l'oscillateur de van der Pol. I O On a trouvé, pour expression de l'opérateur, $H_{\mathrm{op}}\left(a_{t}, p\right)$ :

$$
H_{\mathrm{op}}\left(a_{t}, p\right)=L_{1} C_{1} p^{2}+\left(M s+r_{1} C_{1}+\frac{3 M b}{4} a_{t}^{2}\right) p+\mathrm{I} .
$$

$2^{\circ}$ L'opérateur $H_{\mathrm{op}}\left(a_{t}, j \omega_{0}+q\right)$ est donc de la forme

$L_{1} C_{1}\left(j \omega_{0}+q\right)^{2}+\left(M s+r_{1} C_{1}+\frac{3 M b}{4} a_{t}^{2}\right)\left(j \omega_{0}+q\right)+\mathrm{I}$.

Suivant des notations classiques, nous poserons ici $\left({ }^{3}\right)$ :

$$
\begin{gathered}
\omega_{.}^{2}=\frac{\mathrm{I}}{L_{1} C_{1}}, \quad \varepsilon \omega_{s}=-\frac{M_{s}+r_{1} C_{1}}{2 L_{1} C_{1}}, \\
V_{s}^{2}=-\frac{M_{s}+r_{1} C_{1}}{3 M b} ;
\end{gathered}
$$

$H_{\mathrm{op}}\left(a_{t}, \omega_{0}, q\right)$ devient

$$
\left(j \omega_{0}+q\right)^{2}-2 \varepsilon \omega_{s}\left[\mathrm{I}-\frac{a_{l}^{2}}{4 V_{s}^{2}}\right]\left(j \omega_{0}+q\right)+\omega_{s}^{2} .
$$

$3^{\circ}$ Négligeant les termes en $q^{2}$, ainsi que le produit $\left(\mathrm{I}-\frac{a_{t}^{2}}{4 V_{s}^{2}}\right) q$ si l'on suppose que l'amplitude de la solution synchronisée est voisine de l'amplitude stabilisée, on en tire

$$
|\boldsymbol{H}|^{2}=\left(\omega_{s}^{2}-\omega_{0}^{2}\right)^{2}+\left[2 q \omega_{0}-2 \varepsilon \omega_{s} \omega_{0}\left(\mathrm{I}-\frac{a_{t}^{2}}{4 V_{s}^{2}}\right)\right]^{2} ;
$$

nous utiliserons, conformément aux traités classiques, la valeur réduite $\frac{|\boldsymbol{H}|^{2}}{4 \omega_{0}^{2}}$ et poserons

$$
\Delta \omega=\frac{\omega_{s}^{2}-\omega_{0}^{2}}{2 \omega_{0}} .
$$

$4^{\circ}$ Nous retrouvons l'expression classique de l'équation différentielle du second ordre qui permet d'étudier la stabilité de l'oscillation synchronisée :

$$
\begin{aligned}
& \frac{\mathrm{I}}{4 \omega_{0}^{2}}\left[\mathrm{I}+\alpha_{l}^{2} \frac{\partial}{\partial\left(a_{l}^{2}\right)}\right]_{a_{t}=a_{0}}\left|\boldsymbol{H}\left(a_{\iota}, \omega_{0}, q\right)\right|^{2} \xi \\
& \equiv\left[q^{2}-2 \varepsilon \omega_{0}\left(\mathrm{I}-\frac{a_{0}^{2}}{2 V_{s}^{2}}\right)\right. \\
& \left.\quad+\varepsilon^{2} \omega_{0}^{2}\left(\mathrm{I}-\frac{a_{0}^{2}}{4 V_{s}^{2}}\right)\left(\mathrm{I}-\frac{3 a_{0}^{2}}{4 V_{s}^{2}}\right)+(\Delta \omega)^{2}\right] \xi=0 .
\end{aligned}
$$

Toute cette étude a été poursuivie sous la direction du Professeur P. Grivet qui n'a cessé de me prodiguer ses précieux conseils, orientant constamment mes recherches et mettant à ma disposition tous les documents nécessaires.

Je lui exprime ici ma gratitude la plus vive.

(3) Y. Rocard. Dynamique générale des vibrations.

Manuscrit reçu le 9 juillet ${ }_{9} 5_{2}$. 